Cambridge University Press

978-0-521-84112-2 - Introductory Biomechanics: From Cells to Organisms

C. Ross Ethier and Craig A. Simmons

Frontmatter

More information

\title{
Introductory Biomechanics From Cells to Organisms
}

Introductory Biomechanics is a new, integrated text written specifically for engineering students. It provides a broad overview of this important branch of the rapidly growing field of bioengineering. A wide selection of topics is presented, ranging from the mechanics of single cells to the dynamics of human movement. No prior biological knowledge is assumed and in each chapter, the relevant anatomy and physiology are first described. The biological system is then analyzed from a mechanical viewpoint by reducing it to its essential elements, using the laws of mechanics, and then linking mechanical insights back to biological function. This integrated approach provides students with a deeper understanding of both the mechanics and the biology than that obtained from qualitative study alone. The text is supported by a wealth of illustrations, tables, and examples, a large selection of suitable problems and many current references, making it an essential textbook for any biomechanics course.

C. Ross Ethier is a Professor of Mechanical and Industrial Engineering, the Canada Research Chair in Computational Mechanics, and the Director of the Institute of Biomaterials and Biomedical Engineering at the University of Toronto, with cross-appointment to the Department of Ophthalmology and Vision Sciences. His research focuses on biomechanical factors in glaucoma and on blood flow and mass transfer in the large arteries. He has taught biomechanics for over 10 years.

Craig A. Simmons is the Canada Research Chair in Mechanobiology and an Assistant Professor in the Department of Mechanical and Industrial Engineering at the University of Toronto, with cross-appointments to the Institute of Biomaterials and Biomedical Engineering and the Faculty of Dentistry. His research interests include cell and tissue biomechanics and cell mechanobiology, particularly as it relates to tissue engineering and heart valve disease. 
Cambridge University Press

978-0-521-84112-2 - Introductory Biomechanics: From Cells to Organisms

C. Ross Ethier and Craig A. Simmons

Frontmatter

More information

Cambridge Texts in Biomedical Engineering

Series Editors

W. Mark Saltzman Yale University

Shu Chien University of California, San Diego

Series Advisors

William Hendee Medical College of Wisconsin

Roger Kamm Massachusetts Institute of Technology

Robert Malkin Duke University

Alison Noble Oxford University

Bernhard Palsson University of California, San Diego

Nicholas Peppas University of Texas at Austin

Michael Sefton University of Toronto

George Truskey Duke University

Cheng Zhu Georgia Institute of Technology

Cambridge Texts in Biomedical Engineering provides a forum for high-quality, accessible textbooks targeted at undergraduate and graduate courses in biomedical engineering. It will cover a broad range of biomedical engineering topics from introductory texts to advanced topics including, but not limited to, biomechanics, physiology, biomedical instrumentation, imaging, signals and systems, cell engineering, and bioinformatics. The series will blend theory and practice, aimed primarily at biomedical engineering students but will be suitable for broader courses in engineering, the life sciences and medicine. 


\title{
Introductory Biomechanics
}

\section{From Cells to Organisms}

\author{
C. Ross Ethier and Craig A. Simmons
}

University of Toronto, Canada 
Cambridge University Press

978-0-521-84112-2 - Introductory Biomechanics: From Cells to Organisms

C. Ross Ethier and Craig A. Simmons

Frontmatter

More information

\section{CAMBRIDGE \\ UNIVERSITY PRESS}

University Printing House, Cambridge CB2 8BS, United Kingdom

Cambridge University Press is part of the University of Cambridge.

It furthers the University's mission by disseminating knowledge in the pursuit of education, learning and research at the highest international levels of excellence.

www.cambridge.org

Information on this title: www.cambridge.org/9780521841122

(C) C. R. Ethier and C. A. Simmons 2007

This publication is in copyright. Subject to statutory exception and to the provisions of relevant collective licensing agreements, no reproduction of any part may take place without the written permission of Cambridge University Press.

First published 2007

10th printing 2015

Printed in the United Kingdom by TJ International Ltd, Padstow, Cornwall

A catalog record for this publication is available from the British Library

ISBN 978-0-521-84112-2 Hardback

Cambridge University Press has no responsibility for the persistence or accuracy of URLs for external or third-party internet websites referred to in this publication, and does not guarantee that any content on such websites is, or will remain, accurate or appropriate. 
Cambridge University Press

978-0-521-84112-2 - Introductory Biomechanics: From Cells to Organisms

C. Ross Ethier and Craig A. Simmons

Frontmatter

More information

To my family, who make it all worthwhile.

C. ROSS ETHIER

To Deborah,

and to my parents, who inspired my love of learning.

CRAIG A. SIMMONS 
Cambridge University Press

978-0-521-84112-2 - Introductory Biomechanics: From Cells to Organisms

C. Ross Ethier and Craig A. Simmons

Frontmatter

More information 
Cambridge University Press

978-0-521-84112-2 - Introductory Biomechanics: From Cells to Organisms

C. Ross Ethier and Craig A. Simmons

Frontmatter

More information

\section{Contents}

About the cover

page xii

Preface

1 Introduction

1.1 A brief history of biomechanics 3

1.2 An outline of this book 12

References $\quad 15$

2 Cellular biomechanics 18

2.1 Introduction to eukaryotic cellular architecture 18

2.2 The cell's energy system 22

2.3 Overview of the cytoskeleton 23

2.3.1 Actin filaments $\quad 25$

2.3.2 Intermediate filaments 28

2.3.3 Microtubules 28

2.4 Cell-matrix interactions 29

2.5 Methods to measure the mechanical properties of cells and biomolecules

2.5.1 Atomic force microscopy 35

2.5.2 Optical trapping ("optical tweezers") 41

2.5.3 Magnetic bead microrheometry 42

2.5.4 Micropipette aspiration $\quad 43$

2.6 Models of cellular biomechanical behavior $\quad 53$

2.6.1 Lumped parameter viscoelastic model of the cell 54

2.6.2 Tensegrity model of the cytoskeleton $\quad 60$

$\begin{array}{ll}\text { 2.6.3 Modeling actin filaments as a foam } & 69\end{array}$

2.6.4 Computational model of a chondrocyte in its matrix 72

2.7 Mechanotransduction: how do cells sense and respond to mechanical events? 76

2.7.1 Mechanoreceptors $\quad 76$

$\begin{array}{ll}\text { 2.7.2 Intracellular signal transduction } & 78\end{array}$

2.7.3 Cellular response to mechanical signals $\quad 80$ 
Cambridge University Press

978-0-521-84112-2 - Introductory Biomechanics: From Cells to Organisms

C. Ross Ethier and Craig A. Simmons

Frontmatter

More information

2.8 Techniques for mechanical stimulation of cells 80

2.8.1 Compressive loading 81

2.8.2 Stretching 82

2.8.3 Fluid flow 86

2.9 Summary of mechanobiological effects on cells in selected tissues $\quad 90$

2.9.1 Endothelial cells in the vascular system 91

2.9.2 Smooth muscle cells in vascular tissue 93

2.9.3 Chondrocytes in articular cartilage 94

2.9.4 Osteoblasts and osteocytes in bone 95

2.10 Problems 99

References $\quad 111$

3 Hemodynamics 119

3.1 Blood rheology 119

3.1.1 Blood composition 121

3.1.2 Relationship between blood composition and rheology 124

3.1.3 Constitutive equation for blood 129

$\begin{array}{ll}3.2 & \text { Large artery hemodynamics } \\ & 130\end{array}$

3.2.1 Physical characteristics of blood flow patterns in vivo $\quad 130$

3.2.2 Steady blood flow at low flow rates 133

3.2.3 Unsteady flow in large vessels $\quad 138$

3.3 Blood flow in small vessels 142

3.3.1 Fahraeus-Lindqvist effect 143

3.3.2 "Inverse" Fahraeus-Lindqvist effect 146

$\begin{array}{lll}3.4 & \text { Problems } & 147\end{array}$

$\begin{array}{ll}\text { References } & 161\end{array}$

4 The circulatory system 164

4.1 Anatomy of the vasculature 164

$\begin{array}{lll}4.2 & \text { The heart } & 169\end{array}$

4.2.1 Gross anatomy of the heart 171

4.2.2 Qualitative description of cardiac pumping 172

4.2.3 Cardiac pumping power and ventricular function $\quad 175$

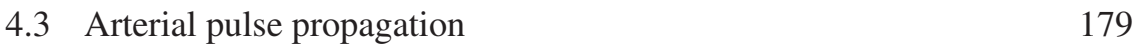

4.3.1 Systolic and diastolic pressure 179

$\begin{array}{ll}\text { 4.3.2 Windkessel model } & 180\end{array}$

4.3.3 Arterial wall structure and elasticity 183

4.3.4 Elastic waves 186

4.3.5 Pressure-flow relationships: purely oscillatory flow 194 
Cambridge University Press

978-0-521-84112-2 - Introductory Biomechanics: From Cells to Organisms

C. Ross Ethier and Craig A. Simmons

Frontmatter

More information

4.3.6 Pressure-flow relationships: mean flow effects

4.3.7 Pressure-flow relationships: deviations from ideality

4.4 The capillaries

4.4.1 Capillary filtration: the experiments of Landis

4.4.2 Osmotic pressure

4.4.3 Quantitative analysis of capillary leakage

4.5 The veins

4.6 Scaling of hemodynamic variables

4.7 Problems

References

5 The interstitium 240

$\begin{array}{lll}5.1 & \text { Interstitial fluid flow } & 240\end{array}$

5.1.1 Darcy's law 241

5.1.2 Clearance of edema $\quad 242$

$\begin{array}{lll}5.2 \text { Problems } & 248\end{array}$

$\begin{array}{ll}\text { References } & 249\end{array}$

6 Ocular biomechanics 250

$\begin{array}{ll}6.1 \text { Ocular anatomy } & 250\end{array}$

$\begin{array}{ll}6.2 \text { Biomechanics of glaucoma } & 251\end{array}$

6.2.1 Tonometry 252

6.2.2 Drainage of aqueous humor in normal and glaucomatous eyes 257

6.2.3 Aqueous humor circulation in the anterior chamber 263

6.2.4 Optic nerve head biomechanics 264

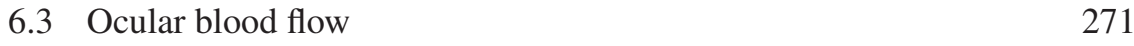

$\begin{array}{lll}6.4 & \text { Problems } & 274\end{array}$

$\begin{array}{ll}\text { References } & 276\end{array}$

7 The respiratory system $\quad 282$

$\begin{array}{lll}7.1 & \text { Gross anatomy } & 282\end{array}$

7.1.1 The conducting airways and pulmonary vasculature 282

7.1.2 Associated structures 285

$\begin{array}{lll}7.2 & \text { Biomechanics of breathing } & 287\end{array}$

7.3 Lung elasticity and surface tension effects 288

$\begin{array}{ll}7.4 \text { Mass transfer } & 294\end{array}$

7.4.1 Blood-side acinar mass transfer $\quad 295$

$\begin{array}{ll}\text { 7.4.2 Air-side acinar mass transfer } & 307\end{array}$

7.4.3 Whole lung mass transfer $\quad 308$

$\begin{array}{lll}7.5 & \text { Particle transport in the lung } & 313\end{array}$ 
Cambridge University Press

978-0-521-84112-2 - Introductory Biomechanics: From Cells to Organisms

C. Ross Ethier and Craig A. Simmons

Frontmatter

More information

7.6 Problems 316

References $\quad 329$

8 Muscles and movement 332

8.1 Skeletal muscle morphology and physiology 333

8.1.1 Isotonic versus isometric contraction 339

8.2 Muscle constitutive modeling 343

$\begin{array}{lll}8.3 & \text { Whole muscle mechanics } & 351\end{array}$

8.3.1 Parallel versus pinnate muscle types 351

8.4 Muscle/bone interactions 353

8.4.1 Foreleg motion in two species 353

$\begin{array}{ll}\text { 8.4.2 Flexion of the elbow } & 355\end{array}$

8.4.3 Biomechanics of the knee $\quad 365$

$\begin{array}{lll}8.5 & \text { Problems } & 369\end{array}$

References 376

9 Skeletal biomechanics 379

$\begin{array}{lll}9.1 & \text { Introduction to bone } & 379\end{array}$

9.2 Composition and structure of bone 382

9.2.1 Cortical bone $\quad 384$

9.2.2 Trabecular bone $\quad 384$

9.3 Biomechanical properties of cortical and trabecular bone 387

9.3.1 Cortical bone mechanics 388

9.3.2 Trabecular bone mechanics $\quad 389$

9.3.3 Trabecular bone mechanics: density dependence $\quad 390$

9.3.4 Trabecular bone mechanics: unit cell models 393

9.4 Bone fracture and failure mechanics 395

$\begin{array}{ll}\text { 9.4.1 Fast fracture } & 397\end{array}$

9.4.2 Fatigue fracture 403

9.5 Functional adaptation and mechanobiology 407

9.6 The design of bone 409

9.7 Introduction to soft connective tissues 411

9.8 Structure of collagen 412

9.9 Structure of ligament, tendon, and cartilage 414

9.9.1 Ligament $\quad 414$

9.9.2 Tendon 416

9.9.3 Cartilage 418

9.10 Biomechanical properties of ligament, tendon, and cartilage 419

9.10.1 Structural properties 420

9.10.2 Material properties $\quad 423$

9.10.3 Material properties: tension $\quad 425$ 
Cambridge University Press

978-0-521-84112-2 - Introductory Biomechanics: From Cells to Organisms

C. Ross Ethier and Craig A. Simmons

Frontmatter

More information

9.10.4 Material properties: compression $\quad 426$

9.10.5 Material properties: viscoelasticity $\quad 428$

9.10.6 Material properties: biphasic mixture theory of cartilage 435

9.11 Problems 436

References $\quad 439$

10 Terrestrial locomotion 444

10.1 Jumping 444

10.1.1 Standing jump 444

10.1.2 Running jumps 448

10.2 Description of walking and running 451

10.2.1 Walking 451

10.2.2 Running 459

10.3 Gait analysis $\quad 461$

10.3.1 Kinematics 463

10.3.2 Anthropometry 468

10.3.3 Kinetics 471

10.4 Problems 480

$\begin{array}{ll}\text { References } & 487\end{array}$

Appendix The electrocardiogram 489

Index 498

Color plate section between pages 118 and 119 
Cambridge University Press

978-0-521-84112-2 - Introductory Biomechanics: From Cells to Organisms

C. Ross Ethier and Craig A. Simmons

Frontmatter

More information

\section{About the cover}

The cover contains images that together represent the broad scope of modern biomechanics. The figures are as follows:

- Main image: A fluorescent immunohistochemical image of an endothelial cell isolated from the surface of a pig aortic heart valve and grown in culture. Within the cell, the nucleus is stained blue and vimentin filaments are stained green. Vimentin is an intermediate filament protein of the cellular cytoskeleton that plays an important role in cellular mechanics.

- Left top: An intermediate stage from a simulation of the forced unfolding of repeats 4 and 5 of chain A of the protein filamin. Filamin is an actin cross-linking protein and therefore plays a role in the biomechanics of the cytoskeleton. The simulation was based on the crystal structure of part of filamin [1], and was carried out in NAMD [2] and visualized using the VMD package [3]. (Image courtesy of Mr. Blake Charlebois.)

- Left middle: A sketch by the Swiss anatomist Hermann von Meyer of the orientation of trabecular bone in the proximal human femur. This sketch was accompanied in the original article by a sketch of the principal stress trajectories in a crane having a shape similar to the femur. Together these sketches are believed to have inspired "Wolff's Law" of bone remodelling. From [4].

- Left lower: The distribution of mass transfer rates from flowing blood to cultured vascular endothelial cells. The contoured quantity (the Sherwood number) was computed by first measuring the topography of the endothelial cells using atomic force microscopy and then solving the convection-diffusion equation in the blood flowing over the cells. Mass transfer from blood to endothelial cells is important in cell-cell signalling. (Image courtesy of Mr. Ji Zhang.)

\section{References}

1. G. M. Popowicz, R. Muller, A. A. Noegel, M. Schleicher, R. Huber and T. A. Holak. Molecular structure of the rod domain of dictyostelium filamin. Journal of Molecular Biology, 342 (2004), 1637-1646. 
Cambridge University Press

978-0-521-84112-2 - Introductory Biomechanics: From Cells to Organisms

C. Ross Ethier and Craig A. Simmons

Frontmatter

More information

2. L. Kale, R. Skeel, M. Bhandarkar, R. Brunner, A. Gursoy, N. Krawetz et al. NAMD2: Greater scalability for parallel molecular dynamics. Journal of Computational Physics, 151 (1999), 283-312.

3. W. Humphrey, A. Dalke and K. Schulten. VMD: Visual Molecular Dynamics. Journal of Molecular Graphics, 14 (1996), 33-38.

4. J. Wolff. Über die innere Architectur der Knochen und ihre Bedeutung für die Frage vom Knochenwachsthum. Archiv für Pathologische Anatomie und Physiologie und für Klinische Medicin, 50 (1870), 389-450. 
Cambridge University Press

978-0-521-84112-2 - Introductory Biomechanics: From Cells to Organisms

C. Ross Ethier and Craig A. Simmons

Frontmatter

More information 
Cambridge University Press

978-0-521-84112-2 - Introductory Biomechanics: From Cells to Organisms

C. Ross Ethier and Craig A. Simmons

Frontmatter

More information

\section{Preface}

For some years, we have taught an introductory course in biomechanics within the Department of Mechanical and Industrial Engineering at the University of Toronto. We have been unable to find a textbook suitable for the purpose of introducing engineers and others having a "hard science" background to the field of biomechanics. That is not to say that excellent books on biomechanics do not exist; in fact, there are many. However, they are typically at a level that is too advanced for an introductory course, or they cover too limited a subset of topics for purposes of an introductory course.

This book represents an attempt to fill this void. It is not meant to be an extensive treatise on any particular branch of biomechanics, but rather to be an introduction to a wide selection of biomechanics-related topics. Our hope is that it will aid the student in his or her introduction to the fascinating world of bioengineering, and will lead some to pursue the topic in greater detail.

In writing this book, we have assumed that the reader has a background in engineering and mathematics, which includes introductory courses in dynamics, statics, fluid mechanics, thermodynamics, and solid mechanics. No prior knowledge of biology, anatomy, or physiology is assumed, and in fact every section begins with a review of the relevant biological background. Each chapter then emphasizes identification and description of the essential aspects of the related biomechanics problems. Because of the introductory nature of this book, this has led in some cases to a great deal of simplification, but in all instances, we have tried to maintain a firm link to "biological reality."

We wish to thank Professor David F. James, of the Department of Mechanical and Industrial Engineering, University of Toronto. He first developed the introductory course in biomechanical engineering at the University and his course notes provided the inspiration for parts of this book. Professors James E. Moore Jr. and Takami Yamaguchi provided important material for Ch. 1. We have benefited greatly from interactions with our students, who sometimes are the best teachers, and our colleagues and mentors.

We shall be most grateful to students who, upon discovering errors in the text, bring them to our attention. 